Medical Research Archives, Volume 5 Issue 6.June Issue.

- Case Report -

\title{
Diffuse Large B-cell Lymphoma Coexistent with Tuberculous Pleurisy Diagnosed by Diagnostic Thoracoscopy
}

\section{Diffuse Large B-cell Lymphoma Coexistent with Tuberculous Pleurisy Diagnosed by Diagnostic Thoracoscopy}

Authors :

Yuri Baba ${ }^{1}$, Yugo Kaneko ${ }^{1}$, Ikumi Fujisaki $^{1}$, Hironori Kawamoto ${ }^{1}$, Hirofumi Yamauchi $^{2}$, Kai Ryu ${ }^{1}$, Nayuta Saito ${ }^{1}$, Sho Watanabe $^{1}$, Tsugumi Horikiri ${ }^{1}$, Yutaka Yoshii $^{3}$, Aya Seki ${ }^{1}$, Akira Kinoshita ${ }^{1}$, Hiroshi Takeda ${ }^{1}$, Keisuke Saito ${ }^{1}$, Kazuyoshi Kuwano 3

\section{Authors note:}

${ }^{1}$ Division of Respiratory Diseases, Department of Internal Medicine, The Jikei University Daisan Hospital, 4-11-1 Izumihoncho, Komae-shi, Tokyo 2018601, Japan

2 Division of Clinical Oncology/Hematology, Department of Internal Medicine, The Jikei University Daisan Hospital, 4-11-1 Izumihoncho, Komae-shi, Tokyo 201-8601, Japan ${ }^{3}$ Division of Respiratory Diseases, Department of Internal Medicine, The Jikei University School of Medicine, 3-258 Nishi-Shimbashi, Minato-ku, Tokyo 105-8461, Japan

\section{Corresponding author:}

Yuri Baba, M.D.

Department of Respiratory Medicine, The Jikei University Daisan Hospital, 4-11-1 Izumihoncho, Komae-shi, Tokyo 2018601, Japan

E-mail:koyuringo42@gmail.com

Phone: +81-3-3480-1151;

Fax: +81-3-3480-6688

\section{Email Addresses :}

Yugo Kaneko, M.D., Ph.D.: yugojax@gmail.com Ikumi Fujisaki,

M.D.: ikumi_64@msn.com

Hironori Kawamoto, M.D.:

h5amm1212@yahoo.co.jp

Hirofumi Yamauchi, M.D.:

h.yamauchi@jikei.ac.jp

Kai Ryu, M.D.: kai5947@yahoo.co.jp

Nayuta Saito, M.D.:

nayutasaito@jikei.ac.jp

Sho Watanabe ,M.D.:

sh.watanabe02@gmail.com Tsugumi

Horikiri, M.D.:

tsugumi.s@world.ocn.ne.jp

Yutaka Yoshii, M.D.:

y.yoshii@jikei.ac.jp

Aya Seki, M.D.: aya-matsu@jikei.ac.jp

Akira Kinoshita, M.D.:

kinoppy@jikei.ac.jp Hiroshi Takeda,

M.D.: inspiron@jikei.ac.jp

Keisuke Saito, M.D., Ph.D.: keisuke@jikei.ac.jp

Kazuyoshi Kuwano, M.D., Ph.D.: kkuwano@jikei.ac.jp 


\title{
Diffuse Large B-cell Lymphoma Coexistent with Tuberculous Pleurisy Diagnosed by Diagnostic Thoracoscopy
}

\begin{abstract}
An 80-year-old man was admitted with massive right pleural effusion and bilateral axillary lymph node enlargement. Because malignant lymphoma was suspected by cytological examination of the pleural effusion, axillary lymph node biopsy was performed. Histopathological examination revealed diffuse large B-cell lymphoma by immunostaining. However, the level of pleural adenosine deaminase (P-ADA) was elevated at 79.2 IU/L and the result of enzyme-linked immunospot assay was positive, although the results of acid-fast staining and polymerase chain reaction (PCR) for Mycobacterium tuberculosis using a pleural effusion sample were both negative. Thus, because the coexistence of tuberculous pleurisy was strongly suspected, diagnostic thoracoscopy was performed under local anesthesia. The diagnostic thoracoscopy revealed multiple white lesions on the parietal pleura. Histopathologic examination showed granuloma including Langhans giant cells with necrosis. Then, because the $M$. tuberculosis culture of the pleural biopsy tissue was positive, tuberculous pleurisy was definitively diagnosed. When the level of P-ADA is elevated in cases of malignant lymphoma, the coexistence of tuberculous pleurisy should be suspected, even though acid-fast staining and PCR might be negative. In such cases, diagnostic thoracoscopy should then be considered to definitively diagnose tuberculous pleurisy.
\end{abstract}

Key words: diffuse large B-cell lymphoma , tuberculous pleurisy , adenosine deaminase, thoracoscopy 
Medical Research Archives, Volume 5 Issue 6.June Issue.

- Case Report -

\section{Diffuse Large B-cell Lymphoma Coexistent with Tuberculous Pleurisy Diagnosed by Diagnostic Thoracoscopy}

\section{Introduction}

There have been several reports related to pleural effusion accompanied by malignant lymphoma. $^{1-4}$ In such cases, malignant lymphoma is usually diagnosed by cytopathologic examination of the pleural effusion sample. Several articles reported that the level of pleural adenosine deaminase (P-ADA) ranged widely from 11 to 98.9 IU/L in cases of malignant lymphoma with pleural effusion. 2,5,6 However, when pleural effusion is predominantly lymphocytic with a high level of P-ADA, tuberculous pleurisy is generally suspected. In the present report, we describe a case of diffuse large B-cell lymphoma (DLBCL) coexistent with tuberculous pleurisy diagnosed with the aid of diagnostic thoracoscopy.

\section{Case Report}

An 80-year-old man with a past medical history of hypertension was admitted to The Jikei University Daisan Hospital due to anorexia and general malaise in April 2014. Laboratory data on admission are shown in Table 1. Although his white blood cell count was within normal range at $4200 / \mu \mathrm{L}$, his Creactive protein level was elevated at 7.0 $\mathrm{mg} / \mathrm{dL}$. His soluble IL-2 receptor level was $2100 \mathrm{U} / \mathrm{mL}$. Chest X-ray revealed a massive right pleural effusion (Fig. 1). Chest contrast-enhanced computed tomography also revealed right pleural effusion and swelling of the mediastinal and the bilateral axillary lymph nodes. The lung field was normal (Fig. 2).

Malignant disease was suspected because of hemorrhagic exudative pleural effusion. Cytological examination of the pleural effusion revealed large-sized atypical round cells with irregular karyotype that suggested malignant lymphoma (Fig. 3). Then, a biopsy of the right axillary lymph node was performed. Histopathological examination of the lymph node revealed diffuse medium-to-large-sized atypical round cells with moderate cytoplasm that were positive for CD20 and CD79 $\alpha$ staining and negative for $\mathrm{CD} 3, \mathrm{CD} 5$, and CD30 staining (Fig. 4). As a result, DLBCL was definitively diagnosed.

Although the results of acid-fast staining and polymerase chain reaction (PCR) analysis of Mycobacterium tuberculosis using the pleural effusion sample, which was predominantly lymphocytic and exudative, were both negative, the P-ADA level was elevated at $79.2 \mathrm{IU} / \mathrm{L}$. Moreover, the result of an enzyme-linked immunospot assay (TSPOT.TB test; Oxford Immunotec Ltd., Summertown, UK) was positive. Because the coexistence of tuberculous pleurisy was suspected from these results, diagnostic thoracoscopy was performed under local anesthesia. Multiple white lesions were seen on the parietal pleura (Fig. 5). Unfortunately, the intrathoracic region could not be sufficiently observed because there were many severe adhesions. No tumors suspected of being malignant lymphoma were noted during the limited observation. Histopathological findings showed granuloma including Langhans giant cells with necrosis (Fig. 6). Because $M$. tuberculosis culture of the pleural tissue biopsied during the thoracoscopy and that of the pleural effusion were positive at 2 weeks and 4 weeks, respectively (Table 2), tuberculous pleurisy was definitively diagnosed. Ultimately, the patient was diagnosed as having both DLBCL and tuberculous pleurisy, although it was unclear whether the relation between the two diseases was as one being the complication of the other or just one of coexistence.

Anti-tuberculosis treatment was started with 3 standard drugs (isoniazid, rifampicin, and ethambutol) 6 days after performing the thoracoscopy because of the patient's advanced age. After the beginning of tuberculous treatment, we started combination chemotherapy with vincristine 


\section{Diffuse Large B-cell Lymphoma Coexistent with Tuberculous Pleurisy Diagnosed by Diagnostic Thoracoscopy}

and cyclophosphamide against DLBCL while paying attention to any worsening of the tuberculosis. These treatments were safely completed without severe adverse events or deterioration of the tuberculosis. After the tuberculosis treatment and chemotherapy, the patient's right pleural effusion had diminished, and swelling of the mediastinal and bilateral axillary lymph nodes was reduced.

\section{Discussion}

In the present case, the measurement of PADA and diagnostic thoracoscopy were useful examinations for diagnosing tuberculous pleurisy, which was suggested by the high P-ADA level. Moreover, granuloma with Langhans giant cells was identified from the pleural tissue biopsied during the thoracoscopy, and the culture of M. tuberculosis from the biopsy tissue was positive.

P-ADA is often measured in cases of pleural effusion and is elevated in various conditions such as malignant lymphoma, empyema, rheumatoid arthritis, and malignant mesothelioma in addition to tuberculous pleurisy. Some cases of malignant lymphoma were also reported that were difficult to distinguish from tuberculous pleurisy based on the level of $\mathrm{P}$ ADA. ${ }^{2,7}$ However, when the cut-off value for P-ADA level was set at $40 \mathrm{IU} / \mathrm{L}$, the sensitivity and specificity of P-ADA for tuberculous pleurisy ranged from $68-91 \%$ and $72-92 \%$, respectively. ${ }^{8-11}$ In contrast, Yao et al. reported that the median level of P-ADA was as low as 26 IU/L in cases of malignant lymphoma with exudative pleural effusion. ${ }^{12}$ Because the level of P-ADA was markedly elevated at $79.2 \mathrm{IU} / \mathrm{L}$ in the present case, the result was compatible with tuberculous pleurisy.
Therefore, we believe that the measurement of P-ADA is useful for auxiliary diagnosis of tuberculous pleurisy to distinguish it from malignant lymphoma.

Tuberculosis sometimes develops alongside hematological malignancies such as malignant lymphoma and leukemia. 13,14 Hence, when chemotherapy for malignant lymphoma is administered, it is crucial to check for the coexistence of tuberculosis. In the present case, because the acid-fast bacillus smear and PCR analysis of the pleural effusion sample were negative, these examinations did not lead to the correct diagnosis. Indeed, in patients with tuberculous pleurisy, the positive rates of acid-fast bacillus smear and culture using pleural effusion samples are low at less than $10 \%$ and $40 \%$, respectively. ${ }^{15,16}$ However, because the elevated level of P-ADA suggested the coexistence of tuberculosis in the present case, diagnostic thoracoscopy was performed under local anesthesia that proved the coexistence of tuberculous pleurisy. Moreover, M. tuberculosis was more rapidly cultured from the pleural biopsy tissue than from the pleural effusion sample, and afterwards, anti- tuberculosis susceptibility testing could be performed. Hence, diagnostic thoracoscopy was useful for diagnosing tuberculous pleurisy.

In conclusion, the measurement of PADA and diagnostic thoracoscopy assisted in the accurate diagnosis of tuberculous pleurisy in a patient with malignant lymphoma. When the level of P-ADA is elevated in cases of malignant lymphoma, the coexistence of tuberculous pleurisy should be suspected, even though acid-fast staining and PCR results might be negative. In such cases, diagnostic thoracoscopy should be considered to definitively diagnose tuberculous pleurisy. 
Medical Research Archives, Volume 5 Issue 6.June Issue.

- Case Report -

\section{Diffuse Large B-cell Lymphoma Coexistent with Tuberculous Pleurisy Diagnosed by Diagnostic Thoracoscopy}

\section{References}

1. Okuda M, Tanaka N, Kashio M, et al. Rapid improvement of massive bloody pleural effusion after splenectomy for splenic marginal zone lymphoma. $B M J$ Case Rep. 2009;2009:bcr0920080974.

2. Kato F, Hirasawa Y, lioka Y, et al. [A case of primary effusion lymphoma with elevation of ADA activity in pleural effusion]. Nihon Kokyuki Gakkai Zasshi. 2011;49(10):786- 791.

3. Bachuwa G, Naik P, Campe J, Lecea N, Congdon D. Ninety-one year old: oldest patient reported with pulmonary mucosa-associated lymphoid tissue lymphoma and rare association with pleural effusion. Geriatr Gerontol Int. 2012;12(1):149-151.

4. Sekiguchi N, Noto S, Wagatsuma M, et al. Extramedullary hematopoietic pleural effusion accompanied by follicular lymphoma. Internal Med. 2013;52(24):2801-2804.

5. Ungerer JP, Grobler SM. Molecular forms of adenosine deaminase in pleural effusions. Enzyme. 1988;40(1):7-13.

6. Ocaña I, Martinez-Vazquez JM, Ribera E, Segura RM, Pascual C. Adenosine deaminase activity in the diagnosis of lymphocytic pleural effusions of tuberculous, neoplastic and lymphomatous origin. Tubercle. 1986;67(2):141-145.

7. Mito A, Ohashi N, Akita S, et al. [A case of natural killer cell lymphoma with high adenosine deaminase level in pleural effusion]. Nihon Kokyuki Gakkai Zasshi. 2005;43(6):360-364.

8. De Oliveira HG, Rossatto ER, Prolla JC. Pleural fluid adenosine deaminase and lymphocyte proportion: clinical usefulness in the diagnosis of tuberculosis. Cytopathology. 1994;5(1):27-32.
9. Pèrez-Rodríguez E, Pérez Walton IJ, Sanchez Hernández JJ, et al. ADA1/ADAp ratio in pleural tuberculosis: an excellent diagnostic parameter in pleural fluid. Respir Med. 1999;93(11):816-821.

10. Lima DM, Colares JK, da Fonseca BA. Combined use of the polymerase chain reaction and detection of adenosine deaminase activity on pleural fluid improves the rate of diagnosis of pleural tuberculosis. Chest. 2003;124(3):909-914.

11. Tahhan M, Ugurman F, Gozu A, Akkalyoncu B, Samurkasoglu B. Tumour necrosis factor-alpha in comparison to adenosine deaminase in tuberculous pleuritis. Respiration. 2003;70(3):270-274.

12. Yao CW, Wu BR, Huang KY, Chen HJ. Adenosine deaminase activity in pleural effusions of lymphoma patients. QJM. 2014;107(11):887-893.

13. Ruiz-Argüelles GJ, Arizpe-Bravo D, Garcés-Eisele J, Sánchez-Sosa S, RuizArgüelles A, Ponce-de-León S. Tuberculosis-associated fatal hemophagocytic syndrome in a patient with lymphoma treated with fludarabine. Leuk Lymphoma. 1998;28(5-6):599- 602.

14. Kindler T, Schindel C, Brass U, Fischer T. Fatal sepsis due to mycobacterium tuberculosis after allogeneic bone marrow transplantation. Bone Marrow Transplant. 2001;27(2):217-218.

15. Epstein DM, Kline LR, Albelda SM, Miller WT. Tuberculous pleural effusions. Chest. 1987;91(1):106-109.

16. Valdés L, Alvarez D, San José E, et al. Tuberculous pleurisy: a study of 254 patients. Arch Intern Med. 1998;158(18):2017-2021. 
Medical Research Archives, Volume 5 Issue 6.June Issue.

- Case Report -

Diffuse Large B-cell Lymphoma Coexistent with Tuberculous Pleurisy Diagnosed by Diagnostic Thoracoscopy

\section{Figure legends}

Figure 1: Chest X-ray on admission revealed marked right pleural effusion.

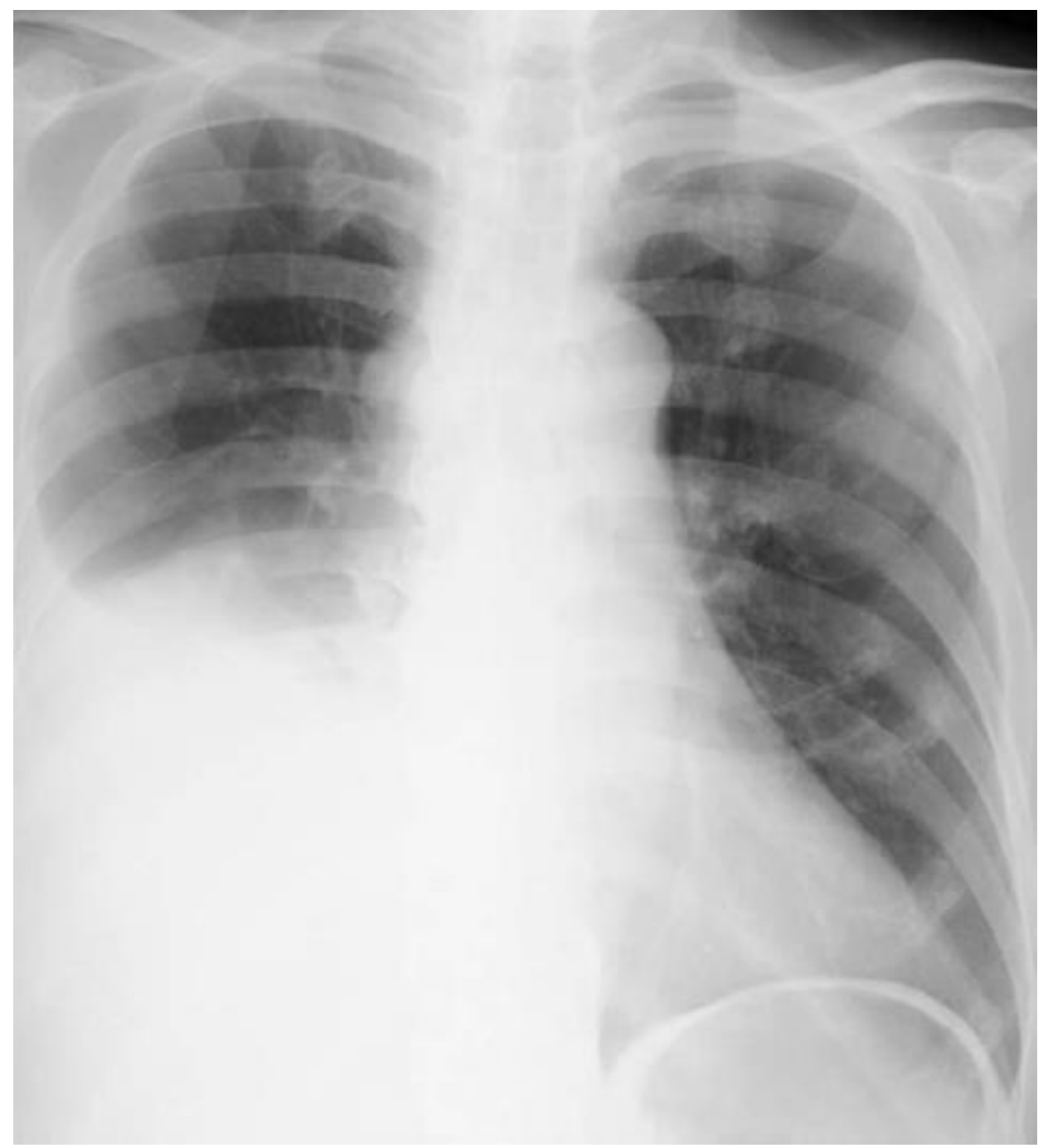


Medical Research Archives, Volume 5 Issue 6.June Issue.

- Case Report -

\section{Diffuse Large B-cell Lymphoma Coexistent with Tuberculous Pleurisy Diagnosed by Diagnostic Thoracoscopy}

Figure 2: Chest contrast-enhanced computed tomography on admission. A-B: Swelling of the mediastinal and right axillary lymph nodes. C: Normal lung field. D: Marked right pleural effusion.

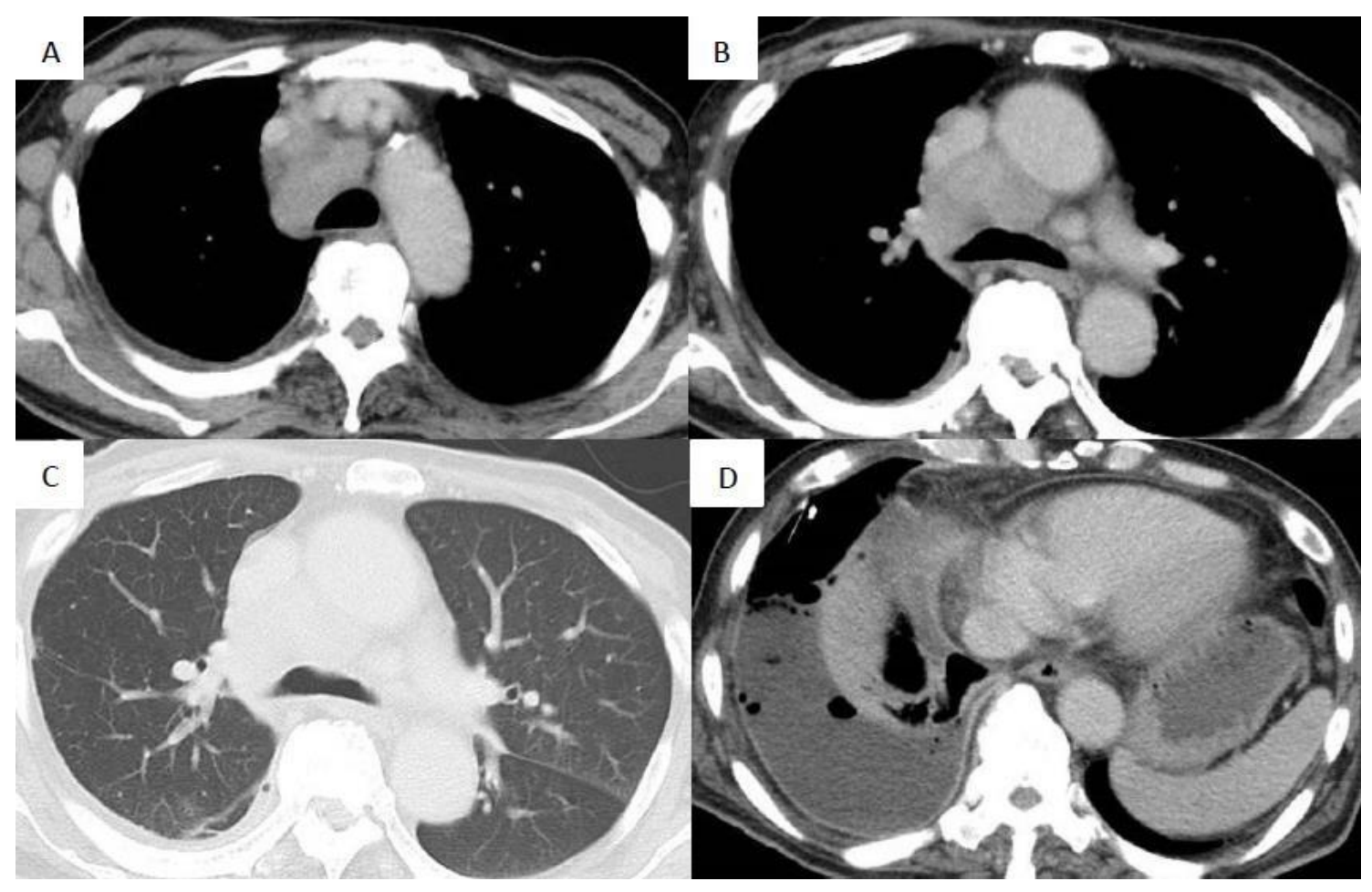


Medical Research Archives, Volume 5 Issue 6.June Issue.

- Case Report -

\section{Diffuse Large B-cell Lymphoma Coexistent with Tuberculous Pleurisy Diagnosed by Diagnostic Thoracoscopy}

Figure 3: Large-sized atypical round cells showing karyotype irregularity were identified in the right pleural effusion (Papanicolaou staining, original magnification $\times 600$ ).

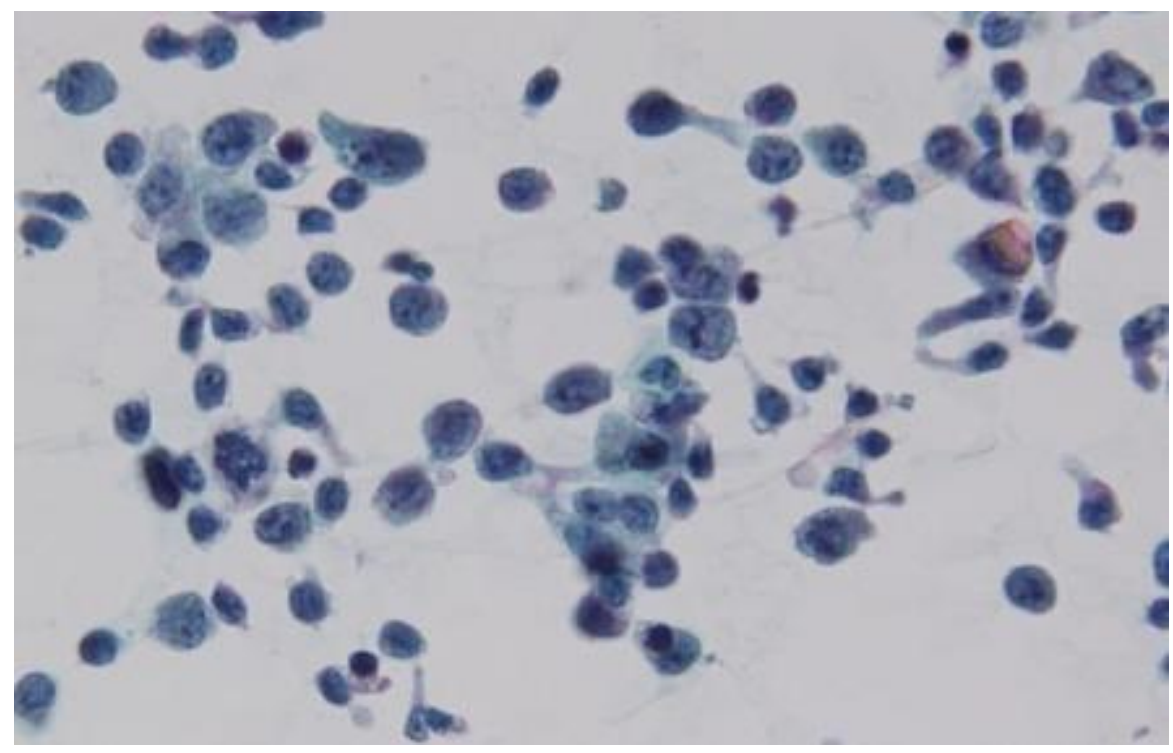


Medical Research Archives, Volume 5 Issue 6.June Issue.

- Case Report -

\section{Diffuse Large B-cell Lymphoma Coexistent with Tuberculous Pleurisy Diagnosed by Diagnostic Thoracoscopy}

Figure 4: Diffuse medium-to-large-sized atypical round cells with moderate cytoplasm were identified in the right axillary lymph node (hematoxylin and eosin staining, original magnification $\times 400)$.

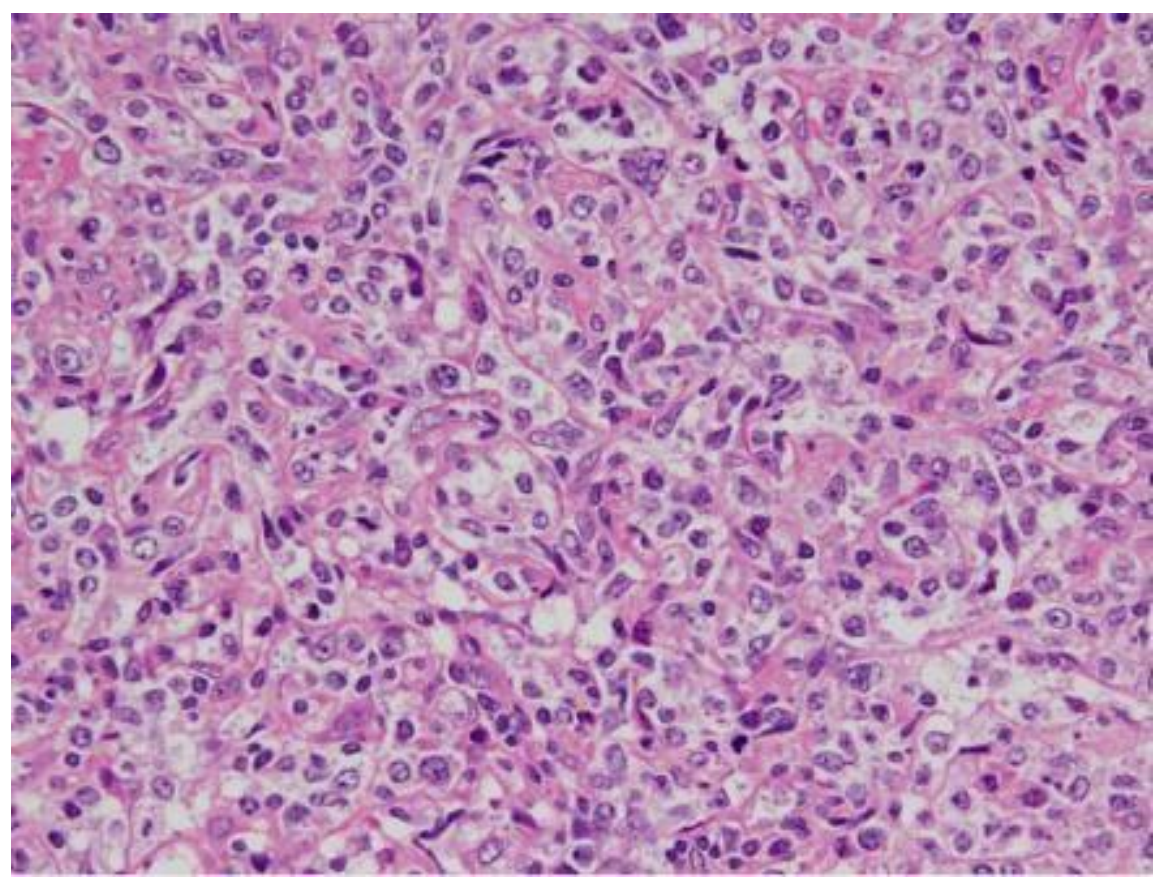


Medical Research Archives, Volume 5 Issue 6.June Issue.

- Case Report -

\section{Diffuse Large B-cell Lymphoma Coexistent with Tuberculous Pleurisy Diagnosed by Diagnostic Thoracoscopy}

Figure 5: Thoracoscopy under local anesthesia showed severe adhesions and multiple white lesions on the parietal pleura.

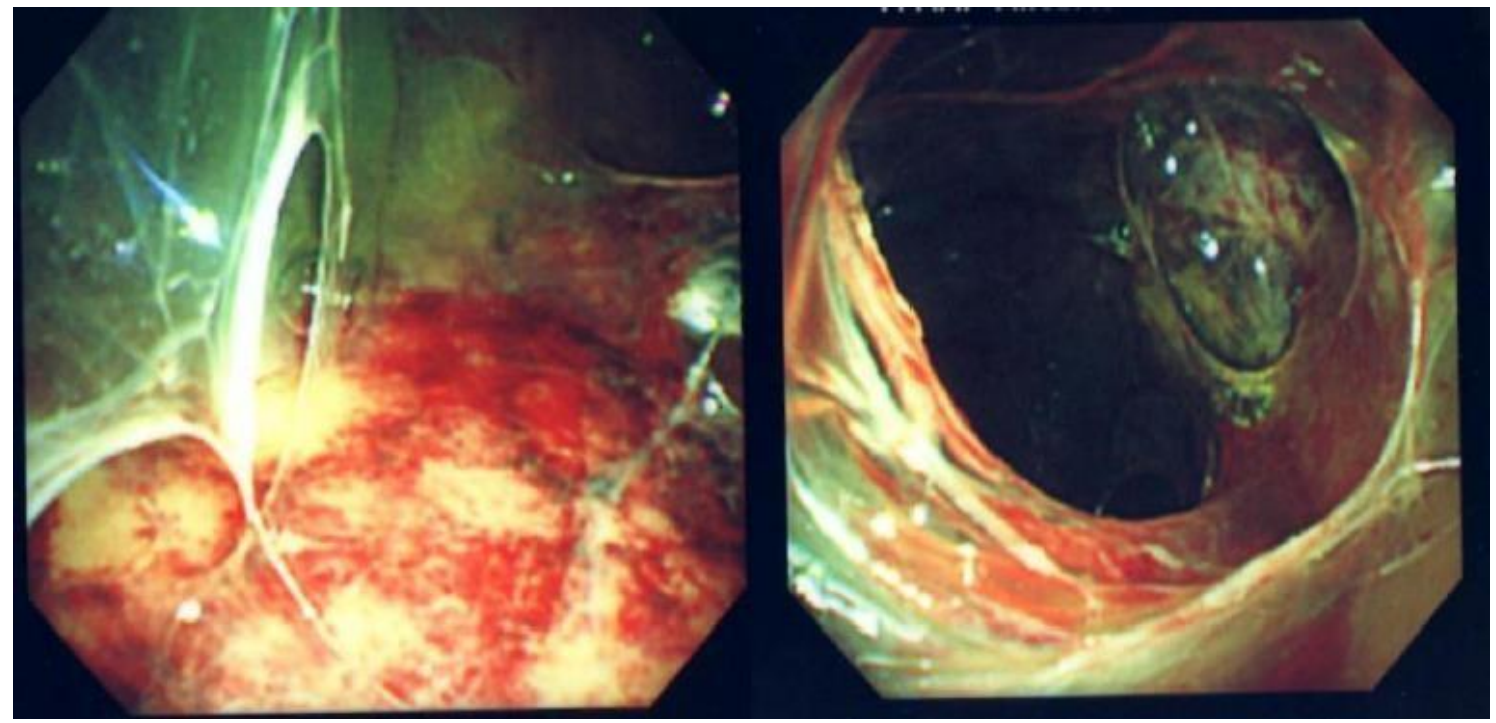


Medical Research Archives, Volume 5 Issue 6.June Issue.

- Case Report -

\section{Diffuse Large B-cell Lymphoma Coexistent with Tuberculous Pleurisy Diagnosed by Diagnostic Thoracoscopy}

Figure 6: Pathological examination of the pleural tissue biopsy specimen revealed (A) Langhans giant cells and (B) granuloma (both, hematoxylin and eosin staining, original magnification $\times 200$ ).
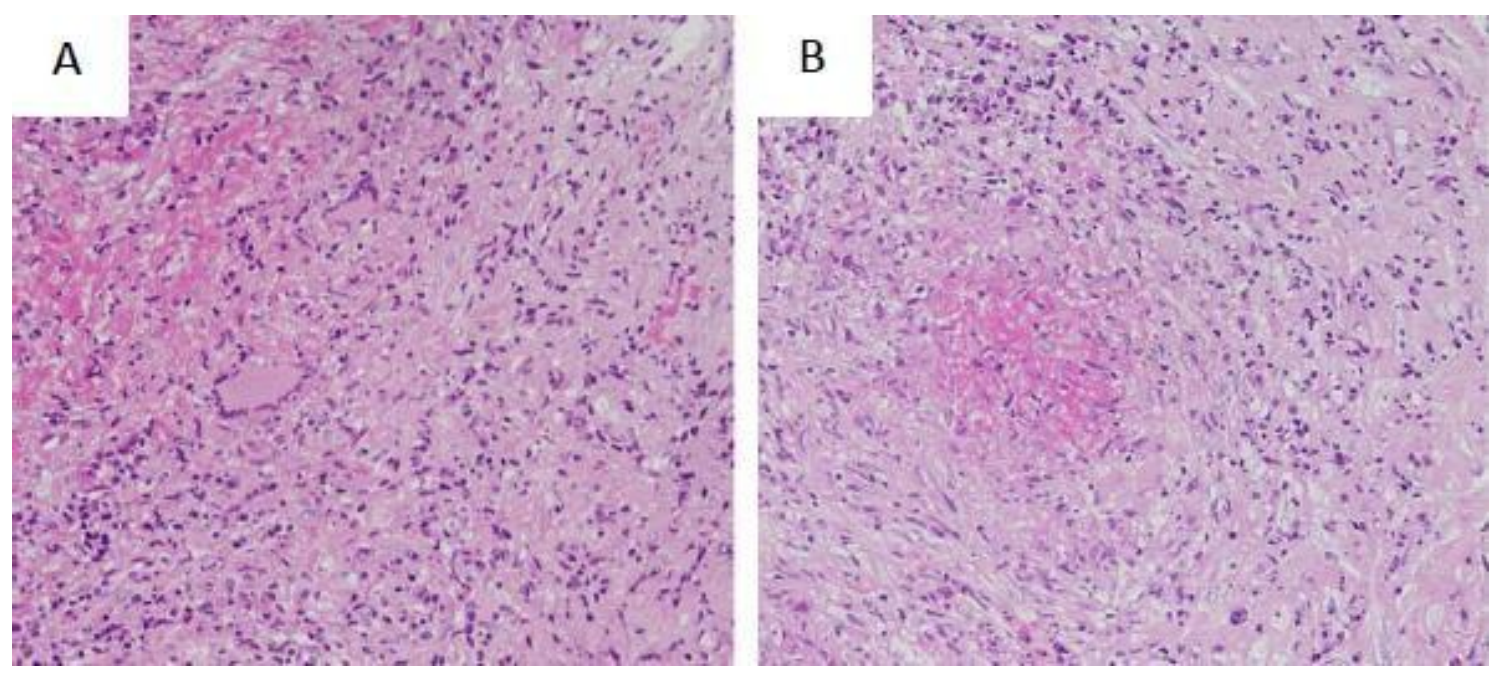
Medical Research Archives, Volume 5 Issue 6.June Issue.

- Case Report -

Diffuse Large B-cell Lymphoma Coexistent with Tuberculous Pleurisy Diagnosed by Diagnostic Thoracoscopy

Table 1. Laboratory Data on Admission

\begin{tabular}{llll}
\hline Peripheral Blood Data & \multicolumn{3}{l}{ Biochemistry Data } \\
\hline White blood cells & $4200 / \mu \mathrm{L}$ & Aspartate aminotransferase & $38 \mathrm{IU} / \mathrm{L}$ \\
Red blood cells & $323 \times 10^{4} / \mu \mathrm{L}$ & Alanine aminotransferase & $17 \mathrm{IU} / \mathrm{L}$ \\
Hemoglobin & $8.7 \mathrm{~g} / \mathrm{dL}$ & Lactate dehydrogenase & $302 \mathrm{IU} / \mathrm{L}$ \\
Hematocrit & $27.2 \%$ & Total protein & $8.2 \mathrm{~g} / \mathrm{dL}$ \\
Platelets & $7.7 \times 10^{4} / \mu \mathrm{L}$ & Albumin & $2.5 \mathrm{~g} / \mathrm{dL}$ \\
& & Blood urea nitrogen & $18 \mathrm{mg} / \mathrm{dL}$ \\
Serology & & Creatinine & $0.9 \mathrm{mg} / \mathrm{dL}$ \\
C-reactive protein & $7 \mathrm{mg} / \mathrm{dL}$ & & \\
Soluble IL-2 receptor & $2100 \mathrm{U} / \mathrm{mL}$ & & \\
Immunoglobulin G & $3192 \mathrm{mg} / \mathrm{dL}$ & & \\
Antinuclear antibody & $40 \mathrm{times}$ & & \\
Rheumatoid factor & $3.0 \mathrm{U} / \mathrm{ml}$ & & \\
T-SPOT.TB test & Positive & & \\
\hline
\end{tabular}


Medical Research Archives, Volume 5 Issue 6.June Issue.

- Case Report -

Diffuse Large B-cell Lymphoma Coexistent with Tuberculous Pleurisy Diagnosed by Diagnostic Thoracoscopy

Table 2. Pleural Effusion and Pleural Tissue Biopsy Data

\begin{tabular}{cllc}
\hline Pleural effusion & \multicolumn{3}{c}{ Pleural tissue biopsy data } \\
\hline Total cell count & $860 / \mu \mathrm{L}$ & Acid-fast bacillus smear & Negative \\
Neutrophil count & $208 / \mu \mathrm{L}$ & TB PCR & Negative \\
Lymphocytes & $652 / \mu \mathrm{L}$ & Mycobacterium culture & Positive at 2 weeks \\
Total protein & $6.1 \mathrm{~g} / \mathrm{dL}$ & Drug resistance & None \\
Lactate dehydrogenase & $397 \mathrm{IU} / \mathrm{L}$ & & \\
Glucose & $51 \mathrm{mg} / \mathrm{dL}$ & & \\
Adenosine deaminase & $79.2 \mathrm{IU} / \mathrm{L}$ & & \\
Cytology & Class V & & \\
Acid-fast bacillus smear & Negative & & \\
TB PCR & Negative & & \\
Mycobacterium culture & Positive at 4 weeks & & \\
Drug resistance & None & & \\
\hline
\end{tabular}

TB: tuberculosis, PCR: polymerase chain reaction. 